\title{
Comparative Analysis of Radiofrequency Ablation and Microwave Ablation for Critically Located Hepatocellular Carcinomas Smaller than $5 \mathrm{~cm}$
}

\author{
Ravindran Ramalingam ${ }^{1 \#}$ Amar Mukund ${ }^{1, \oplus \#}$ Karan Manoj Anandpara ${ }^{1} \quad$ Yashwant Patidar $^{1}$ \\ Shiv Kumar Sarin ${ }^{2}$ \\ ${ }^{1}$ Department of Interventional Radiology, Institute of Liver and \\ Biliary Sciences, New Delhi, India \\ ${ }^{2}$ Department of Hepatology, Institute of Liver and Biliary Sciences,

\begin{abstract}
Address for correspondence Amar Mukund, MD, Department of Radiology, Institute of Liver and Biliary Sciences, D-1, Vasant Kunj, New Delhi-110070, India (e-mail: dramarmukund@gmail.com).
\end{abstract} New Delhi, India

J Clin Interv Radiol ISVIR

\begin{abstract}
Purpose To compare the safety and efficacy of radiofrequency ablation (RFA) versus microwave ablation (MWA) for hepatocellular carcinomas (HCC) smaller than $5 \mathrm{~cm}$ in critical locations.

Methods Single-center retrospective study of all patients who underwent RFA/ MWA for HCC from July 2015 to Dec 2019. Critical location includes exophytic tumors, tumors $\leq 5 \mathrm{~mm}$ from the diaphragm, heart, gallbladder, kidney, gastrointestinal tract, and $\leq 10 \mathrm{~mm}$ from large vessels with caliber of $\geq 3 \mathrm{~mm}$. Treatment effectiveness, local tumor progression, and complication rates were evaluated.

Results Out of 119 patients with $147 \mathrm{HCC}$ nodules in critical location, 65 (M: $\mathrm{F}=49: 16$; mean age-61.7) were included in RFA group and 54 (M:F =43:11; mean age-60.5) in MWA group. Mean follow-up period was 16.5 and 14.8 months, respectively. At first follow-up imaging, 66/78 tumors in RFA group and 57/69 tumors in MWA group showed complete ablation with primary treatment effectiveness rates of $84.6 \%$ and $82.6 \%$, respectively $(p=0.741)$. Local tumor progression (LTP) rate was $21.8 \%(17 / 78)$ and $20.3 \%(14 / 69)$, respectively $(p=0.826)$. Median time to LTP was 12 and 13.5 months, respectively. Fourteen tumors in RFA group and 12 in MWA group underwent reab-

Keywords

- carcinoma

- hepatocellular

- radiofrequency ablation

- microwave ablation lation with a secondary treatment effectiveness rates of $78.6 \%(14 / 17)$ and $83.3 \%$ $(12 / 14)$, respectively $(p=0.757)$. Mean LTP-free survival was 37.2 and 28.1 months, respectively. The total complication rate was $36.9 \%$ and $31.5 \%$, respectively $(p=0.535)$ with no major complications in both the groups.

Conclusion Our data suggest that both MWA and RFA are equally safe and effective for treating $\mathrm{HCCs}<5 \mathrm{~cm}$ in critical locations.
\end{abstract}

\section{Introduction}

Hepatocellular carcinoma (HCC) is the sixth most common cancer. HCC has been recognized as a leading cause of death among patients with chronic liver disease (CLD) and has been found to have a higher morbidity in Asian countries than the Western world. Although surgical resection and liver transplantation are considered as curative treatment options for BCLC stage 0 (very early stage) and stage A (early stage) HCCs, ${ }^{1}$ more than $80 \%$ of patients are not amenable to

(c) 2021. Indian Society of Vascular and Interventional Radiology. This is an open access article published by Thieme under the terms of the Creative Commons Attribution-NonDerivative-NonCommercial-License, permitting copying and reproduction so long as the original work is given appropriate credit. Contents may not be used for commercial purposes, or adapted, remixed, transformed or built upon. (https://creativecommons.org/licenses/by-nc-nd/4.0/).

Thieme Medical and Scientific Publishers Pvt. Ltd. A-12, 2nd Floor, Sector 2, Noida-201301 UP, India 
surgery. Majority of these cases are managed with percutaneous ablation therapies, which can be as effective as surgical techniques in terms of disease-free survival and overall survival. ${ }^{2-4}$

Radiofrequency ablation (RFA) is the most common ablation technique used globally and is considered as safe and effective curative modality for treatment of HCC $<3 \mathrm{~cm} .{ }^{5}$ But RFA is generally risky when the tumor is subcapsular or located within $1 \mathrm{~cm}$ from the common bile duct or adjacent to visceral organs due to increased risk of complications. ${ }^{6-9}$ Also, the efficacy of RFA is less when the tumor lies adjacent to large vessels due to heat sink effect. However, with recent advances in technology, safety and efficacy of RFA in these critical locations should be re-evaluated.

Microwave ablation (MWA) is another thermal ablative technique which uses electromagnetic fields for tissue destruction unlike electrical field in the RFA. MWA has several theoretical advantages over RFA in terms of its ability to achieve steady temperatures above $100^{\circ} \mathrm{C}$, achieves larger ablation volumes in a relatively short period of time, less affected by heat sink and charring effects, and does not require grounding pads unlike monopolar RFA. ${ }^{10,11}$ MWA has gradually replaced RFA at many centers specifically for treating these tumors in a critical location considering its advantages. Despite these advantages, the superiority of MWA over RFA in ablation of liver tumors is still under debate. Although several meta-analyses have shown that both RFA and MWA have similar safety and efficacy profiles, the aim of the present study is to compare the safety and efficacy of RFA versus MWA for HCCs in critical locations.

\section{Materials and Methods}

This retrospective study was approved by the institutional scientific review board and ethical committee. Informed consent was waived off, considering the retrospective nature of the study. Clinical case records of all patients who underwent RFA or MWA for HCC from July 2015 to December 2019 were analyzed. The study included patients who underwent either RFA or MWA for HCCs $<5 \mathrm{~cm}$ and tumor in critical locations. Critical location included exophytic tumors, tumors $\leq 5 \mathrm{~mm}$ from the diaphragm, heart, gallbladder, kidney, gastrointestinal tract, and $\leq 10 \mathrm{~mm}$ from large vessels with caliber of $\geq 3 \mathrm{~mm}$. Patients were explained about both the ablative techniques, their advantages, and cost. Lesions closer to a vessel of diameter $\geq 3 \mathrm{~mm}$ were counselled for MWA and exophytic lesions closer to colon were preferred for RFA. However, patients were left free to decide, depending on their affordability. Patients who underwent combined transcatheter arterial chemoembolization (TACE) plus ablation, those with HCCs $>5 \mathrm{~cm}$, tumors located elsewhere, those who were lost to follow-up, and those with incomplete medical records were excluded from the study. Baseline characteristics of patients age, sex, child score, model for end-stage liver disease (MELD) Na score, serum $\alpha$-fetoprotein, liver function test, maximum tumor diameter, and mean duration of follow-up were recorded and compared between the groups.

\section{RFA Procedure}

All RFA procedures were performed using 15G multi-tinned expandable monopolar RFA electrode (StarBurst Talon RFA electrode for $<3 \mathrm{~cm}$ ablation zone, StarBurst XL RFA electrode for $3-5 \mathrm{~cm}$ ablation zone and StarBurst Xli-enhanced RFA electrode for 4-7 cm ablation zone, AngioDynamics) and RF generator (RITA 1500X RF generator, AngioDynamics). Ablation was performed with a target temperature of $105^{\circ} \mathrm{C}$ and power of $150 \mathrm{~W}$ for 8 minutes.

\section{MWA Procedure}

All MWA procedures were performed using an 15G MWA SOLERO applicator with an internally cooled system and the SOLERO microwave generator with an operating frequency of $2.45 \mathrm{GHz}$ and powers of up to $140 \mathrm{~W}$ (AngioDynamics). Once the applicator was positioned within the tumor, ablation was performed with appropriate power and time based on manufacturer prescribed guidelines.

All RFA and MWA procedures were performed percutaneously under ultrasound (US) guidance ( - Figs. 1 and 2) except for two HCC nodules in the RFA group and one HCC nodule in the MWA group, where additional CT guidance was used, owing to poor localization of tumor by US. All RFA and MWA procedures were performed by a single interventional radiologist with more than 10 years of experience in percutaneous ablation. The procedure was performed under conscious sedation and local anesthesia along with monitoring of vital signs. Ablation zone was monitored continuously under
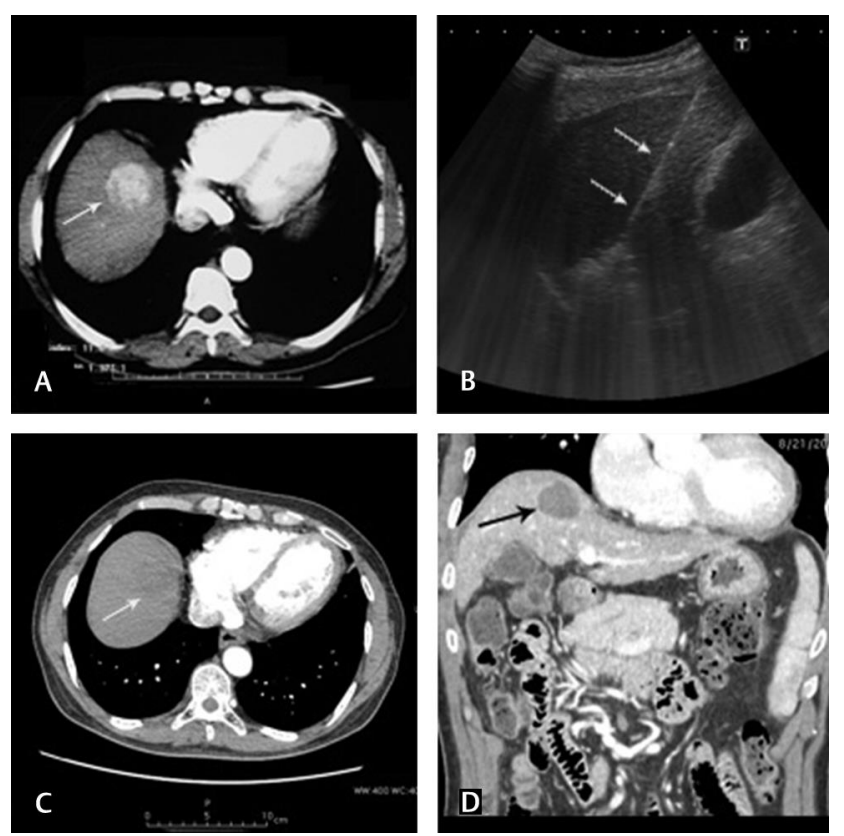

Fig. 1 A 62-year-old man with hepatocellular carcinoma (HCC). (A) Contrast-enhanced CT image shows well-circumscribed arterial enhancing tumor (arrow) in segment VIII located just beneath the diaphragm; (B) Ultrasound (US) image shows radiofrequency ablation (RFA) needle (arrows) placed within the tumor through angled intercostal approach; (C, D). Follow-up contrast-enhanced CT after 1 month in arterial and delayed phase imaging shows no enhancement within the tumor bed (arrow) consistent with complete response. 

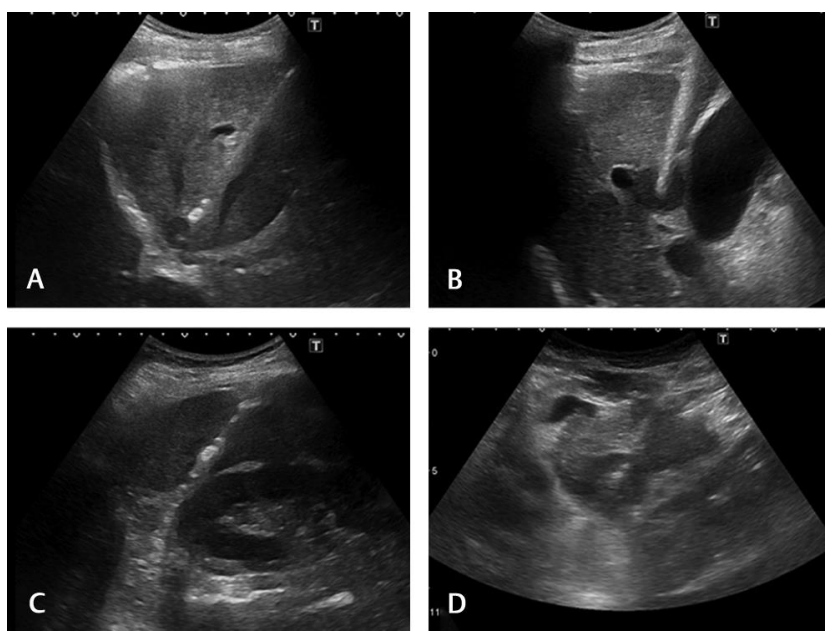

Fig. 2 Ultrasound (US) images show (A) well-defined hypoechoic tumor located between right and middle hepatic vein with microwave ablation (MWA) needle placed parallel to hepatic veins; (B) Well-defined hypoechoic tumor located adjacent to the gall bladder with radiofrequency ablation (RFA) needle placed parallel to and away from gall bladder; (C) Tumor in subnephric location with MWA needle placed away from the kidney; (D) RFA needle placed obliquely into the tumor with intervening normal parenchyma for tumor in exophytic location.

real-time US guidance to ascertain the adequacy of ablation (visualizing an echogenic cloud while ablating due to vapor produced) and screen for any complication. Ablation was considered to be complete if the air cloud covered the entire tumor with at least 5 to $10 \mathrm{~mm}$ of normal adjoining liver parenchyma. In case the ablation zone was found to be inadequate, the needle was repositioned to complete the ablation.

Different strategies were followed for needle placement, depending on the tumor location. Angled intercostal approach was used for subdiaphragmatic tumors ( - Fig. 1). For tumors adjacent to large vessels, the needle tract was planned parallel to the blood vessel (-Fig. 2) to avoid direct injury. Exophytic and subcapsular tumors were approached indirectly with

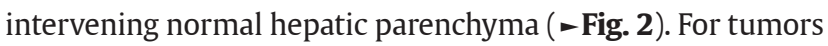
adjacent to visceral organs, needle tract was planned either parallel ( - Fig. 2) or away from the visceral organ, maximizing the distance as much as possible. Tract ablation was performed during needle removal to avoid peritoneal bleeding from capsular surface and tumoral seeding. Hydrodissection was used only for two patients having exophytic lesion indenting over hepatic flexure/colon. Lesions close to the gall bladder were separated by injecting 15 to $20 \mathrm{~mL}$ of dextrose in the plane between lesion and gallbladder to create edema for separating these structures. No other maneuver was used for lesions at other locations.

\section{Posttreatment Assessment and Follow-up}

All patients were screened with US 1 and four hours after the procedure, to look for any complication, along with overnight monitoring of vitals with management of postprocedure pain. The complete blood cell count, liver function tests (LFT), and US of the abdomen were performed the next day, and the patients were discharged in case of tests within normal limits. All patients were followed-up periodically until death or time of data analysis (June 2020). The follow-up included assessment of serum AFP level, LFT, and a contrast-enhanced CT/MRI 1 month after the procedure. The imaging evaluation was done using modified response evaluation criteria in solid tumors (m-RECIST) criteria. Complete response was indicated by the absence of any enhancing area in the tumor bed with an ablative margin of $5 \mathrm{~mm}$ and ablation was said to be incomplete if the nodular enhancing component is still visualized at the tumor site. A repeat ablation or alternative treatment was performed for incompletely ablated tumors, while periodic follow-up was planned for patients having a complete response. The imaging follow-up was repeated every 3 months till 1 year and every 6 months thereafter for assessment of local tumor progression, intra- and extrahepatic recurrences. The intra- or extrahepatic recurrence were treated with ablation or other therapies and monitoring of baseline lesion was continued according to the study design until documentation of local progression or death.

\section{Outcome Measures}

Technical success, primary treatment effectiveness, secondary treatment effectiveness, and local tumor progression rate were defined according to the image-guided tumor ablation: standardization of terminology and reporting criteria. ${ }^{12}$ Technical success was defined as the percentage of tumors which show complete coverage by ultrasound immediately after the procedure. Primary treatment effectiveness (PTE) rate was defined as the percentage of tumors showing complete response at imaging follow-up performed 1 month after ablation. Local tumor progression (LTP) was defined as the appearance of enhancing tumor foci at the edge of ablation zone after at least one contrast enhanced follow-up CT/MRI had documented complete response. Secondary treatment effectiveness (STE) rate was defined as the percentage of tumors that have undergone successful repeat ablation following identification of local tumor progression. New intrahepatic lesions treated with ablation were considered as another index lesion and followed from that point of time for local tumor progression. Procedure-related complications were classified based on adverse event classification by the Society of Interventional Radiology. ${ }^{13}$ The major complication was defined as an event that leads to substantial morbidity and disability, increasing the level of care, or results in hospital admission or substantially lengthened hospital stay. All other complications were regarded as minor.

\section{Statistical Analysis}

All statistical analysis was performed using SPSS v.22.0 (IBM Corp.). The comparison between baseline characteristics of two groups were made either by using Fisher's exact test for a categorically variable data or the Mann-Whitney for a continuous variable data, and characteristics were expressed as mean \pm standard deviation or median with interquartile range. Outcome measures between the two groups were compared 
4 Comparative Analysis of Radiofrequency Ablation and Microwave Ablation Ramalingam et al.

using Pearson's Chi-square test or Z test, where appropriate. A $p$ value of $<0.05$ was considered statistically significant.

\section{RESULTS}

\section{Clinical characteristics}

The study period included 258 patients with 376 HCC tumor nodules treated with either RFA or MWA. Of these, 119 patients with 147 HCC tumor nodules were in critical locations. All the patients had underlying cirrhosis. The common causes of cirrhosis were ethanol abuse, hepatitis B virus (HBV) and hepatitis C virus (HCV). Of 119 patients, 23 were classified as Barcelona clinic liver cancer (BCLC) stage $0,71 / 119$ as stage $A, 20 / 119$ as stage $B$, and 5/119 as stage D. The Eastern Cooperative Oncology Group (ECOG) performance status score was either 0 or 1 for all patients. As per Child-Turcotte-Pugh (CTP) scoring system, 79/119 patients were classified as class A, 35/119 under class B, and 5/119 under class $C$, with CTP scores ranging between 5 to 11. MELD Na score ranging between 8 to 27 .

Sixty-five patients with 78 HCC nodules in critical location were treated with RFA and 54 patients with 69 HCC nodules were treated with MWA. Location of the tumors in RFA and MWA group are described in - Table 1. There was no statistical significance between the groups in the baseline characteristics of patient's age, sex, CTP score, MELD Na score, serum bilirubin, aspartate transaminase (AST), alanine transaminase (ALT) levels, maximum tumor diameter, and follow-up period as outlined in -Tables $\mathbf{2}$ and 3.

Technical Success and Primary Treatment Effectiveness The technical success of $100 \%$ was obtained in both the groups. Sixty-six of 78 tumors in RFA group and 57 of 69 tumors in MWA group showed complete response at first

Table 1 Tumor location in RFA versus MWA group

\begin{tabular}{|l|l|l|l|}
\hline Tumor location & $\begin{array}{l}\text { RFA } \\
\text { group } \\
(\boldsymbol{n}=\mathbf{7 8})\end{array}$ & $\begin{array}{l}\text { MWA } \\
\text { group } \\
(\boldsymbol{n}=\mathbf{6 9 )}\end{array}$ & $p$-Value \\
\hline Near diaphragm & 26 & 14 & 0.08 \\
\hline Near large vessel & 17 & 23 & 0.12 \\
\hline Exophytic/subcapsular & 13 & 6 & 0.15 \\
\hline Near kidney & 9 & 9 & 0.78 \\
\hline Near gall bladder & 7 & 8 & 0.60 \\
\hline $\begin{array}{l}\text { Near gastrointestinal } \\
\text { tract }\end{array}$ & 4 & 6 & 0.39 \\
\hline Near heart & 2 & 3 & 0.55 \\
\hline
\end{tabular}

Abbreviations: MWA, microwave ablation; RFA, radiofrequency ablation.

Table 2 Baseline characteristics of study population

\begin{tabular}{|l|l|l|l|}
\hline Characteristics & $\begin{array}{l}\text { RFA group } \\
(\boldsymbol{n}=65)\end{array}$ & $\begin{array}{l}\text { MWA group } \\
(\boldsymbol{n}=54)\end{array}$ & \multirow{2}{*}{0.427} \\
\hline Age (in years) & & $60.5 \pm 6.9$ & \\
\hline Mean \pm SD & $61.7 \pm 9.1$ & $45-77$ & 0.582 \\
\hline Range & $46-79$ & $43 / 11$ & \\
\hline Sex (male/female) & $49 / 16$ & $21 / 15 / 11 / 7$ & 0.320 \\
\hline Etiology (alcohol/HBV/HCV/others) & $27 / 17 / 13 / 8$ & $7.6 \pm 1.4$ & 0.404 \\
\hline CTP score (mean \pm SD) & $7.9 \pm 1.8$ & $14.4 \pm 6.1$ & 0.218 \\
\hline MELD Na score (mean \pm SD) & $15.3 \pm 5.6$ & $14.5 \pm 86.3$ & 0.113 \\
\hline S. Alpha-fetoprotein (median \pm IQR, ng/ml) & $17.9 \pm 65.8$ & $1.6 \pm 0.5$ & 0.105 \\
\hline S. bilirubin (mean \pm SD, mg/dl) & $1.4 \pm 0.8$ & $52.3 \pm 23.7$ & 0.261 \\
\hline S. aspartate transaminase (mean \pm SD, IU/L) & $59.8 \pm 25.9$ & $43.2 \pm 16.8$ & \\
\hline S. alanine transaminase (mean \pm SD, IU/L) & $47.5 \pm 23.4$ & & \\
\hline
\end{tabular}

Abbreviations: CTP, Child-Turcotte-Pugh; HBV, hepatitis B virus; HCV, hepatitis C virus; IQR, interquartile range; MELD, model for end-stage liver disease; MWA, microwave ablation; RFA, radiofrequency ablation; SD, standard deviation.

Table 3 Baseline tumor characteristics

\begin{tabular}{|l|l|l|l|}
\hline Characteristics & $\begin{array}{l}\text { RFA group } \\
(\boldsymbol{n}=\mathbf{7 8})\end{array}$ & $\begin{array}{l}\text { MWA group } \\
(\boldsymbol{n}=69)\end{array}$ & \multirow{2}{*}{0.139} \\
\hline Maximum tumor diameter (mean \pm SD cm) & $2.7 \pm 0.9$ & $2.9 \pm 0.7$ & 0.082 \\
\hline Largest tumor $(3 \mathrm{~cm} />3 \mathrm{~cm})$ & $71 / 7$ & $56 / 13$ & 0.288 \\
\cline { 1 - 3 } Follow-up (in months) & & & \\
\cline { 1 - 3 } Mean \pm SD & $16.5 \pm 10.1$ & $14.8 \pm 9.1$ & \\
\hline Range & $1-54$ & $1-36$ & \\
\hline
\end{tabular}

Abbreviations: MWA, microwave ablation; RFA, radiofrequency ablation; SD, standard deviation. 
follow-up imaging study with PTE rates of $84.6 \%$ and $82.6 \%$, respectively. This difference was not statistically significant $(p=0.741)$ ( - Table 4$)$. Twelve out of 78 tumors in RFA group and 12/69 tumors in MWA group showed partial response at first follow-up imaging. Of these, 6 tumors in RFA group and 8 tumors in MWA group underwent repeat ablation with complete response at next follow-up imaging. Rest of the tumors with partial response were treated with TACE $(n=4)$, TACE plus ablation $(n=3)$, liver transplantation $(n=1)$, and two were followed-up on conservative measures. Subgroup analysis was performed to compare the results of RFA versus MWA for subcapsular/exophytic tumors, and tumors located adjacent to large vessels and diaphragm. Primary treatment effectiveness for RFA and MWA was not significantly different for subcapsular/exophytic tumors (2/13 vs. $2 / 6, p=0.39$ ), tumors located adjacent to large vessels (4/17 vs. $3 / 23$, $p=0.39)$ and diaphragm (3/26 vs. $3 / 14, p=0.40)$.

\section{Local Tumor Progression and Secondary Treatment Effectiveness}

The mean follow-up period was 16.5 months in the RFA group (range 1-54 months) and 14.8 months in MWA group (range 1-36 months). During the follow-up period till data analysis, LTP was seen in 17 of 78 tumors (21.8\%) in the RFA group and 14 of 69 tumors (20.3\%) in the MWA group ( $p=0.826$ ) ( - Table 4 ). Of these, 14 tumors in RFA group and 10 tumors in MWA group underwent repeat ablation. Rest were treated with TACE $(n=3)$ and liver transplantation $(n=2)$. Median time to local tumor progression was 12 months for RFA group and 13.5 months for MWA group with $p$ value of 0.45 .

Eleven out of 14 tumors in the RFA group and 10 out of 12 tumors in MWA group showed complete response in subsequent follow-up imaging with STE rates of $78.6 \%$ and $83.3 \%$, respectively ( $p=0.757$ ). Rest of these tumors showed partial response in follow-up imaging and were subsequently treated with TACE $(n=3)$, surgical resection $(n=1)$, and liver transplantation $(n=1)$. There was no significant difference in the LTP and STE rates between the groups $(p>0.05)$. LTP for RFA and MWA was also comparable for subcapsular/exophytic tumors (3/13 vs. $2 / 6, p=0.63)$, tumors located adjacent to large vessels $(4 / 17$ vs. $4 / 23, p=0.64)$, and diaphragm (5/26 vs. $3 / 14, p=0.87)$.

Mean estimate of LTP-free survival was 37.2 months (95\% confidence interval: 30.0-44.4) for RFA group and 28.1 months (95\% confidence interval: 24.8-31.5) for
MWA group. LTP-free survival was comparable and statistically not significant between the two groups as depicted by the Kaplan-Meier graph analysis ( $p=0.97,-$ Fig. 3 ).

\section{Complications}

There were no major complications or procedure-related deaths in either group. Three patients (4.6\%) in the RFA group and two patients $(3.7 \%)$ in MWA group had intraperitoneal bleeding which was managed conservatively (minor adverse event, SIR class B). These tumors were located adjacent to diaphragm ( $n=1)$, large vessel $(n=1)$ and exophytic location $(n=1)$ in RFA group and adjacent to diaphragm $(n=1)$ and exophytic location $(n=1)$ in MWA group. Six patients in the RFA group and eight patients in MWA group had mild asymptomatic pleural effusion (minor adverse event, SIR class A), owing to tumor location just beneath the diaphragm and all these were managed conservatively with spontaneous resolution of the same in subsequent follow-up imaging. Fever of $>$ $38^{\circ} \mathrm{C}$ was present in 15 patients in the RFA group (23.1\%) and seven patients in MWA group (13\%), which persisted for 2 to 5 days (minor adverse event, SIR class B). There was no difference in total complication rate between RFA and MWA groups (36.9\% vs. $31.5 \%, p=0.535$ ) (-Table 4). Total complication rate for RFA and MWA was also not significantly different for subcapsular/exophytic tumors (4/13 vs. $2 / 6, p=0.91$ ), tumors

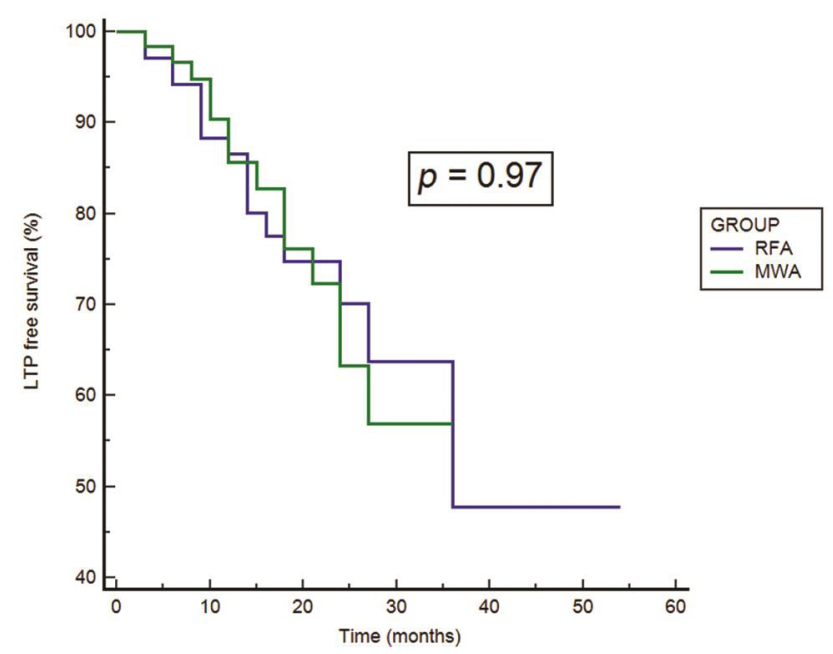

Fig. 3 Kaplan-Meier analysis comparing local tumor progression free survival between radiofrequency ablation (RFA) and microwave ablation (MWA) groups.

Table 4 Outcome measures

\begin{tabular}{|l|l|l|l|}
\hline Characteristics & $\begin{array}{l}\text { RFA group } \\
(\mathrm{Np}=65) \\
(\mathrm{Nt}=78)\end{array}$ & $\begin{array}{l}\text { MWA group } \\
(\mathrm{Np}=54) \\
(\mathrm{Nt}=69)\end{array}$ & $p$-Value \\
\hline PTE rate & $84.6 \%(66 / 78)$ & $82.6 \%(57 / 69)$ & 0.741 \\
\hline LTP rate & $21.8 \%(17 / 78)$ & $20.3 \%(14 / 69)$ & 0.826 \\
\hline STE rate & $78.6 \%(11 / 14)$ & $83.3 \%(10 / 12)$ & 0.757 \\
\hline Total complication rate & $36.9 \%(24 / 65)$ & $31.5 \%(17 / 54)$ & 0.535 \\
\hline
\end{tabular}

Abbreviations: LTP, local tumor progression; Np, number of patients; Nt, number of tumor nodules; PTE, primary treatment effectiveness; STE, secondary treatment effectiveness. 
located adjacent to large vessels (5/17 vs. $2 / 23, p=0.09)$, and diaphragm (10/26 vs. $11 / 14, p=0.12)$. There were no reports of burns at the needle insertion site, tumor seeding, pneumothorax, diaphragmatic hernia or palsy, liver abscess formation, or liver failure.

\section{Discussion}

Percutaneous tumor ablation is one of the most commonly used nonsurgical methods for treating very early stage and early stage HCC.,14 RFA and MWA are the two most commonly used ablative techniques globally, with each having its own merits and demerits. RFA uses electrical current in radiofrequency range which is delivered through a needle electrode. This produces slow and steady rise in temperature in the range of 60 to $100^{\circ} \mathrm{C}$, resulting in coagulative necrosis. But RFA is limited by heat sink and charring effects..$^{15}$ On the other hand, MWA uses electromagnetic energy to create a rapid and homogeneous heating of tissue, ultimately resulting in coagulative necrosis with large ablation volume and shorter ablation times. ${ }^{10,11}$ The advantage of MWA can also be its demerit, as rapid heating of tissues in MWA can easily injure the adjacent critical structures.

Traditionally, RFA is considered risky for tumors at the critical location due to higher incidence of major complications, needle-tract seeding, and local recurrence. In recent years, evolution in RFA devices and technology such as development of multitinned and expandable electrodes, cooled-wet electrodes, and bipolar electrodes have enabled the treatment of HCCs in critical location which were previously not eligible for RFA treatment. From then, RFA for treatment of tumors in critical location has gained importance. Yang et $\mathrm{al}^{16}$ published a retrospective study which included 382 patients in difficult location group and compared with 88 patients in normal location group. The study showed a no significant differences in PTE rates and LTP-free survival rates but LTP rate and complication rates were significantly different between the groups. However, the outcomes of RFA rather needs to be compared with MWA to effectively choose between the two commonly available modalities.

MWA has gradually replaced RFA at many centers specifically for treating these tumors in a critical location considering its theoretical advantages of shorter ablation times with large ablation volumes and less heat sink and charring effects. In the present study, RFA was compared with MWA for tumors in critical locations. The study showed that PTE and STE, LTP, complication and LTP-free survival were comparable between the groups. This might be attributed to the individualized protocols adopted for both RFA and MWA, based on tumor location.

MWA is generally preferred for tumors adjacent to large vessels to reduce heat sink effect, ${ }^{11}$ while RFA is preferred for subcapsular/exophytic lesions adjacent to vital structures as rapid heating in MWA might cause inadvertent organ injury. ${ }^{17}$ Subgroup analysis of present study showed that PTE, LTP, and complication rates were not significantly different between RFA and MWA for both tumors adjacent to large vessels and subcapsular/exophytic tumors.

Meta-analysis by Tan et $\mathrm{l}^{18}$ showed MWA has similar therapeutic effects compared with RFA for treatment of HCCs with no significant differences in complete ablation, local recurrence, disease-free survival, overall survival and major complication rates. Violi et $\mathrm{al}^{19}$ published another randomized controlled phase 2 trial comparing RFA versus MWA and concluded that MWA was not more effective than RFA for treatment of HCCs smaller than $4 \mathrm{~cm}$. Based on the findings of these studies, RFA can be preferred for smaller HCCs, considering its cost advantage. But another recent meta-analysis demonstrated LTP was significantly reduced by $37 \%$ with MWA as compared with RFA among patients with tumor size $\geq 2.5 \mathrm{~cm} .{ }^{20}$ The majority of the tumors included in our study was $<3 \mathrm{~cm}$. So, the advantage of MWA over RFA for ablation of large tumors might be masked in the present study, and further, large randomized controlled trials are needed to prove this effect.

The major complication rate of RFA and MWA remains controversial. The results of previous studies showed nonsignificant trend toward higher complication rate for MWA as compared with RFA. ${ }^{21,22}$ Larger ablation volumes achieved with MWA might increase the risk of damaging neighboring structures, especially vascular and biliary structures accounting for higher complication rates as compared with RFA. But the present study, in fact, showed a lower complication rate with MWA when compared with RFA, with no statistically significant difference between the two. Adjuvant techniques like artificial ascites, ${ }^{23}$ artificial pleural effusion, balloon interposition, or contrast-enhanced US $^{24}$ can be used to further reduce the complication rates, but this was not evaluated in the study.

This study had some limitations. The first being the retrospective nature and smaller sample size. Second, all MWA and RFA were performed with single system operating at a specific frequency, and results varied across systems due to the differences in type of electrode used and differences between generated versus delivered energy. ${ }^{25}$

\section{Conclusion}

Both percutaneous RFA and MWA are equally safe and effective for treatment of small HCCs at critical locations, provided proper patient selection, appropriate preprocedure planning and meticulous technique is all that is needed for complication-free clinical success. Comparison between MWA and RFA still needs to be evaluated further by randomized trials encompassing larger sample size and including an adequate number of tumors of size larger than $3 \mathrm{~cm}$.

\section{Funding}

None.

\section{IRB Statement}

The study was performed in accordance with the principles of the Declaration of Helsinki, and the Institutional Review Board approved this retrospective study. 


\section{Informed Consent}

The Institutional Review Board approved this retrospective study and waived the requirement of informed consent.

\section{Consent for Publication}

Consent for publication was obtained for every individual person's data included in the study.

\section{Conflict of Interest}

None declared.

\section{References}

1 European Association for The Study Of The Liver; European Organisation For Research And Treatment Of Cancer. EASL-EORTC clinical practice guidelines: management of hepatocellular carcinoma. J Hepatol 2012;56(4):908-943

2 Gupta P, Kalra N, Keshava SN, et al. Indian Society of Vascular and Interventional Radiology Expert Consensus Statements for Ablation in Hepatocellular Carcinoma: Part I. J Clin Interv Radiol ISVIR. J Clin Interv Radiol 2020;4(2):98-106

3 Cho YK, Rhim H, Noh S. Radiofrequency ablation versus surgical resection as primary treatment of hepatocellular carcinoma meeting the Milan criteria: a systematic review. J Gastroenterol Hepatol 2011;26(9):1354-1360

4 Feng K, Yan J, Li X, et al. A randomized controlled trial of radiofrequency ablation and surgical resection in the treatment of small hepatocellular carcinoma. J Hepatol 2012;57(4):794-802

5 Mukund A, Vats P, Jindal A, Patidar Y, Sarin SK. Early hepatocellular carcinoma treated by radiofrequency ablation-mid- and long-term outcomes. J Clin Exp Hepatol 2020 (e-pub ahead of print). doi: https://doi.org/10.1016/j.jceh.2020.04.016

6 Livraghi T, Solbiati L, Meloni MF, Gazelle GS, Halpern EF, Goldberg SN. Treatment of focal liver tumors with percutaneous radio-frequency ablation: complications encountered in a multicenter study. Radiology 2003;226(2):441-451

7 Rhim H, Yoon KH, Lee JM, et al. Major complications after radio-frequency thermal ablation of hepatic tumors: spectrum of imaging findings. Radiographics 2003;23(1):123-134, discussion 134-136

8 Meloni MF, Goldberg SN, Moser V, Piazza G, Livraghi T. Colonic perforation and abscess following radiofrequency ablation treatment of hepatoma. Eur J Ultrasound 2002;15(1-2):73-76

9 Mulier S, Mulier P, Ni Y, et al. Complications of radiofrequency coagulation of liver tumours. Br J Surg 2002;89(10):1206-1222

10 Lu MD, Xu HX, Xie XY, et al. Percutaneous microwave and radiofrequency ablation for hepatocellular carcinoma: a retrospective comparative study. J Gastroenterol 2005;40(11):1054-1060

11 Skinner MG, Iizuka MN, Kolios MC, Sherar MD. A theoretical comparison of energy sources-microwave, ultrasound and laserfor interstitial thermal therapy. Phys Med Biol 1998;43(12): 3535-3547

12 Ahmed M, Solbiati L, Brace CL, et al; International Working Group on Image-guided Tumor Ablation; Interventional
Oncology Sans Frontières Expert Panel; Technology Assessment Committee of the Society of Interventional Radiology; Standard of Practice Committee of the Cardiovascular and Interventional Radiological Society of Europe. Image-guided tumor ablation: standardization of terminology and reporting criteria-a 10-year update. Radiology 2014;273(1):241-260

13 Sacks D, McClenny TE, Cardella JF, Lewis CA. Society of Interventional Radiology clinical practice guidelines. J Vasc Interv Radiol 2003;14(9 Pt 2) :S199-S202

14 Fong Y, Sun RL, Jarnagin W, Blumgart LH. An analysis of 412 cases of hepatocellular carcinoma at a Western center. Ann Surg 1999;229(6):790-799, discussion 799-800

15 Lu DS, Raman SS, Limanond P, et al. Influence of large peritumoral vessels on outcome of radiofrequency ablation of liver tumors. J Vasc Interv Radiol 2003;14(10):1267-1274

16 YangW,YanK,WuGX,etal.Radiofrequencyablation of hepatocellular carcinoma in difficult locations: Strategies and long-term outcomes. World J Gastroenterol 2015;21(5):1554-1566

17 Poulou LS, Botsa E, Thanou I, Ziakas PD, Thanos L. Percutaneous microwave ablation vs radiofrequency ablation in the treatment of hepatocellular carcinoma. World J Hepatol 2015;7(8):1054-1063

18 Tan W, Deng Q, Lin S, Wang Y, Xu G. Comparison of microwave ablation and radiofrequency ablation for hepatocellular carcinoma: a systematic review and meta-analysis. Int J Hyperthermia 2019;36(1):264-272

19 Vietti Violi N, Duran R, Guiu B, et al. Efficacy of microwave ablation versus radiofrequency ablation for the treatment of hepatocellular carcinoma in patients with chronic liver disease: a randomised controlled phase 2 trial. Lancet Gastroenterol Hepatol 2018;3(5):317-325

20 Glassberg MB, Ghosh S, Clymer JW, et al. Microwave ablation compared with radiofrequency ablation for treatment of hepatocellular carcinoma and liver metastases: a systematic review and meta-analysis. OncoTargets Ther 2019;12:6407-6438

21 Ohmoto K, Yoshioka N, Tomiyama Y, et al. Comparison of therapeutic effects between radiofrequency ablation and percutaneous microwave coagulation therapy for small hepatocellular carcinomas. J Gastroenterol Hepatol 2009;24(2):223-227

22 Zhang L, Wang N, Shen Q, Cheng W, Qian GJ. Therapeutic efficacy of percutaneous radiofrequency ablation versus microwave ablation for hepatocellular carcinoma. PLoS One 2013; 8(10):e76119

23 Song I, Rhim H, Lim HK, Kim YS, Choi D. Percutaneous radiofrequency ablation of hepatocellular carcinoma abutting the diaphragm and gastrointestinal tracts with the use of artificial ascites: safety and technical efficacy in 143 patients. Eur Radiol 2009;19(11):2630-2640

24 Rajesh S, Mukund A, Arora A, Jain D, Sarin SK. Contrast-enhanced US-guided radiofrequency ablation of hepatocellular carcinoma. J Vasc Interv Radiol 2013;24(8):1235-1240

25 Ruiter SJ, Heerink WJ, de Jong KP. Liver microwave ablation: a systematic review of various FDA-approved systems. Eur Radiol 2019;29(8):4026-4035 